\title{
The Pragmatic School of Thought in Open Science: A Case Study of Multi- stakeholder Participation in Shaping the Future of Internet Governance
}

\author{
Stephen McCarthy \\ University College \\ Cork \\ Stephen.McCarthy \\ @ucc.ie
}

\author{
Carolanne Mahony \\ University College \\ Cork \\ carolanne.mahony@ \\ ucc.ie
}

\author{
Wendy Rowan \\ University College \\ Cork \\ wendy.rowan@ \\ $\underline{\text { ucc.ie }}$
}

\author{
Huy Tran-Karcher \\ Missions Publiques \\ Huy.Tran- \\ Karcher@missionsp \\ ubliques.com
}

\author{
Manon Potet \\ Missions Publiques \\ manon.potet@missi \\ onspubliques.com
}

\begin{abstract}
The internet is a disruptive technology that continues to define our modern world. However, numerous ethical challenges remain for internet governance going forward, e.g. surveillance capitalism, terrorism and radicalisation. The 'pragmatic' school of thought in open science advocates for collaboration between diverse stakeholder groups (e.g. citizens, academics, practitioners, policymakers) to ensure an informed, and positive imprint for change. However, our understanding of how open science can be used for assimilating knowledge on complex socio-political issues remains nascent. To address this gap, we present findings from 'We, the Internet', a global consultation project which utilised open science practices such as stakeholder-led evaluations and open access publications to engage stakeholders in dialogue around the future of internet governance. Our findings discuss emergent themes on the future of internet governance, and highlight the potential of open science to mobilise groups and combat public scepticism in policy-making.
\end{abstract}

\section{Introduction}

"The internet is the apotheosis of the Pragmatic revolution [in open science], bringing together radical empiricism and democratisation of information in community practice." [1, pg. 1412]

Over the last 40 years, the internet has transformed human relationships and society as we know it. Recent statistics suggest that nearly $59 \%$ of the world's population are now connected to the internet, with high growth rates projected in developing nations going forward [2]. This multiplication of networks has brought with it opportunities for individuals to connect almost instantaneously with friends, family, co-workers and other social groups across the world. The internet also offers an open resource for education and innovation, allowing individuals and entrepreneurs to draw on a treasure trove of online content for upskilling, awareness building, and new product development $[3,4$, 5]. Upcoming developments such as Artificial Intelligence bring an astonishing range of further opportunities for industries, governments, and societies worldwide including the automation of tasks (e.g. Chatbots for customer service [6]) and augmentation of knowledge work (e.g. clinical decision support [7]).

However, despite these technological and societal advances, numerous ethical challenges remain for internet governance going forward. Firstly, significant concerns have been raised around the integrity of information provided over the web, given the rise of 'fake news' and the distribution of disinformation and misinformation to potentially vulnerable groups $[8,9]$. In addition, much of this digitalisation of life has been brought about by large companies who seek to connect people via internet platforms for commercialisation purposes. In particular, the profit motives of these companies have driven the emergence of 'surveillance capitalism' [10], where internet platforms are used to track citizens' activities online with the objective of profiling and influencing behaviour, e.g. Cambridge Analytica. This has raised new concerns around data privacy and security in the digital age, and the meanings we place on our digital identity $[11,12]$. Political decisions are, therefore, urgently needed to steer the future of internet governance in a more responsible, ethical, and inclusive direction. This requires the voice of numerous stakeholders (e.g. citizens, academics, policymakers) to be heard across the world, to ensure an informed, and positive imprint for change [13].

Open science practices provide a means of engaging diverse groups in research and policymaking $[14,15,16$, 17]. The primary objective of open science is to encourage more equitable and transparent collaboration. In particular, the 'pragmatic' school of thought in open science centres on how collaboration between multiple stakeholder groups can make knowledge-creation more 
effective and efficient [14]. Indeed the internet is a core technology enabling this transformation of open science, as stated by Nielsen [18, pg. 111]: "We need to imagine a world where the construction of the scientific information commons has come to fruition. This is a world where all scientific knowledge has been made available online". Pragmatic open science aims to foster knowledge-creation through interactions between scientists and other stakeholder groups [14]. This fosters networked science, where open collaboration is used as a means to transform how we understand the world and how science is conducted [18].

However, our understanding of how open science can be used for tackling socio-political issues such as the future of internet governance remains nascent. In this paper, we take steps in this direction by addressing the following research question: How can open science practices be used to explore socio-political issues of public concern? To explore this question, we draw on case study findings from 'We, the Internet', a global consultation project which seeks stakeholders' thoughts and feelings on internet governance using participation events and forecasting methods. This input will help to shape this technology for a better future, with the support of a strategic network of partners such as the United Nations, European Commission, World Economic Forum, Wikimedia Foundation, and Google. The primary aim of the project is to explore the importance of internet governance and different perspectives on the digitalisation of life. Questions which will be explored include: (i) How should the Internet be managed and governed? (ii) What is the role of the different actors in this interdependent system? (iii) What will be the impact of emerging technologies such as Artificial Intelligence on internet governance? We extend this dialogue to include not only scientists, but also wider stakeholder groups of citizens, practitioners, and policymakers [14].

Based on our case study findings, we contribute insights into how open science practices can harness the power of collectives such as stakeholder groups who can become the new pioneers of evolved and ethical technology use. In particular, we report on stakeholderled evaluations of different models of internet governance proposed by experts. The views of stakeholders will later be shared with strategic partners such as the United Nations and help combat public scepticism in policymaking. We assert that open science can yield valuable insights in shaping a digital policy for the future and explore broad questions for debate such as "what do we want our technology interaction legacy to look like?" Internet governance is not a trivial question to be relegated to the domain of 'armchair experts' and large companies, but a global issue demanding engagement from all members of society.

\section{Background}

\subsection{The Future of Internet Governance}

The rapid adoption of advanced technologies suggests we are progressing towards a new posthumanist era, where the lines between technology and the human race are becoming increasingly blurred [11]. With the development of Artificial Intelligence, Machine Learning, and connected 'smart' devices, there is immense potential to realise significant improvements in all walks of life: from the use of decision tools for complex processes to the creation of assisted living robots for people with chronic health conditions. The internet is a core technology enabling the rapid transformation of human life and represents a complex socio-technical network of people, systems and information [19].

The use of this technology is also intimately linked to our social and psychological being [11, 13]. For instance, many experience the internet as both a benediction and a malediction: while it provides a useful resource for connecting with others and obtaining information, internet addiction is an increasingly widespread phenomenon where individuals stress that they cannot live without it [20]. In addition, this digitalisation has transformed what we believe, how we think, feel and act: the most extreme case of this being when terrorist groups can connect and radicalise citizens using internet platforms. Our engagement with this medium can, therefore impact and be impacted by our attitudes and behaviour. At times we find ourselves misusing technology, which can lead to unintended negative consequences, e.g. smartphone use while driving which increases the level of risk taken [20]. This is reinforced by poor self-regulation and a failure to comply with laws prohibiting such practices.

IT ethics concerns the study of how humans select between technology features, rejecting the misaligned use, or disengaging where there is an assessment of little value. However, 'learning to swim' in this ocean of new technologies does not come instinctively; in general, we undertake lessons to master this skill. The same applies to our use of technology: we must learn how our engagement with technology increasingly shapes the reality we experience - for better or for worse. Nevertheless, to date, questions around the use of technology for beneficial purposes has often been sidestepped in lieu of our fetish for innovation. Instead, such questions are left to the individual's own ethical decision-making process on how to regulate their use of technology in the wider world. Is it time to call for a change? Do we, as a society, want to learn how to engage with technology in a more informed manner? 
After decades of relative light intervention to enable the internet to flourish more freely in its infancy, decision-makers globally and the general public have become increasingly aware of the imperative for more stringent regulation in order to continue harnessing the benefits while containing the drawbacks. It is therefore increasingly urgent that different stakeholder groups have their voices heard on the next steps for this incredible technology. A key question occurs - are we in need of new approaches for educating how to use technology for more ethical and beneficial purposes? The next subsection looks at how open science practice can help provide insights into this question.

\subsection{Open Science Practice}

In recent years, 'openness' has become an increasingly important topic for information systems research and practice. The term 'openness' can be defined as a lack of restriction or boundaries in participation (i.e. egalitarian), transparency and accountability in decision-making (i.e. meritocratic), and receptiveness to change in processes (i.e. selforganising) [21, 22]. Openness is an embedded feature of areas such as: open innovation [4, 5], open data [23], and open-source software $[24,25]$, to name but a few.

Open science is another core component of the openness philosophy which aims to embed equality of participation and transparency in scientific research [14, $15,16,17]$. Open science can be viewed either in a topdown or a bottom-up approach. From a top-down perspective, open science is focused on making scientific research more easily accessible to a broader range of stakeholder groups [17]. From the bottom-up approach, open science can be viewed as collaborations between researchers and members of the public. This perspective is epitomised in the pragmatic school of thought in open science, which aims to make research easier to access, easier to participate in, and more inclusive [14]. Stakeholders are empowered by the opportunity to participate in research.

Examples of open science practices include the use of open citizen dialogues on policymaking issues (e.g. CIMULACT project), citizen engagement in hypothesis-driven research (e.g. Project PigeonWatch), and volunteer mapping and monitoring of a research area (e.g. British Trust for Ornithology).

To make open science more collaborative, online communication tools can be used to support knowledge creation and dialogue. Tacke [26] sees the internet as an open door for practising collaborative research, breaking down traditional barriers, encouraging diversity and inclusion by harnessing the "wisdom of the crowds". The internet has enabled the hosting of social media platforms such as Twitter, Facebook,
Instagram, which are open to stakeholders for selfexpression. Studies suggest that there is an everincreasing thirst for the distribution of knowledge via social media [27]. These are also viewed as vital ingredients for the recruitment of citizens in research and the 'soundbite' reporting of open science findings. Social media is also well placed for global community self-organisation and the promotion of bottom-up open science activities [15].

Open science raises the question of whether we are doing science for people, or doing science with people? Science communication can be a dialogue that involves all aspects of the research process and can make a practical scientific impact on a broader policy level [28]. Our understanding of open science for developing policy, however, is only emerging with several factors yet to be considered, e.g. the fit between research purpose and project design, technology use, the quality of the data, and whether the resulting findings stand up to scrutiny [29]. Nevertheless, there is potential for open science to enable democratic dialogue and futureoriented decision-making through stakeholder participation.

\subsection{Citizen Science and Participation}

Citizen science (CS) is a type of open movement which encourages participation in science from diverse populations [30]. CS democratises science which can help concerned communities to create data to influence policy and promote political decision-making [31]. CS is valued by politicians throughout Europe as a method for creating socially relevant research [32].

CS and multi-stakeholder participation are important partners in the rethinking of how science and the public engage with each other $[14,16]$. CS changes the way science is conducted by involving different stakeholders (e.g. public, academics, practitioners, policymakers) throughout the research process, e.g. idea generation, conduction of research, and dissemination of findings. CS is about inclusiveness and transparency in research be it data, publications, the evaluation of science or the resultant policies driven by this science. The imperative for inclusiveness and transparency means that multistakeholder participation is a driver for open science.

Stakeholder participation relies on deliberative methods, backed by a vast amount of research from all around the world, ranging from political sciences to sociology, from neurosciences to psychology and from communication to philosophy. Collective deliberation is an ancient motive for political thought: the "ability to participate in deliberative or judiciary power" defines the work of Aristotle and the theories on communicative rationality and consensus decision-making of the philosopher Jürgen Habermas [33]. French philosopher 
Bernard Manin [34] also discussed the importance of diverse group deliberation where "the rules can ... be legitimate only as long as they arise from the will of all and represent the will of all". In a nutshell, deliberation groups deliver informed, non-volatile and argumentative opinions. They are said to suffer less polarisation and have a much lower level of volatility than any other channel of opinion gathering.

Participatory design offers one means of engaging a broad range of stakeholders in research, innovation, and public policy decisions. Participatory design aims to increase stakeholder involvement and exposes decisionmakers to a wider variety of perspectives, requirements, and potential solutions [35]. Stakeholder participation efforts can, in turn, lead to increased interest and participation in the democratic process and contribute to a more scientifically literate society [35]. Participatory design comes from the perspective that those who are impacted by a system should have a say in how it is designed [36, 37]. It emerged from the 'Scandinavian approach' of the '60s and '70s which was concerned with the shifting of power dynamics in the workplace due to the introduction of information systems [38, 39]. The aim is to bring together different stakeholders to collectively shape a better future [40]. This is achieved by using a range of different practices, which involve working directly with stakeholders. As stated by Dalsgaard [41, p. 37] participatory design centres on "concerns and values that connect existing techniques, and that are vital and malleable enough to embrace new challenges and inform new techniques".

\begin{tabular}{|l|l|}
\hline Principle & Definition \\
\hline $\begin{array}{l}\text { Decentral- } \\
\text { isation }\end{array}$ & $\begin{array}{l}\text { No permission is required from a central } \\
\text { authority. Implies freedom from } \\
\text { indiscriminate censorship and surveillance. }\end{array}$ \\
\hline $\begin{array}{l}\text { Non- } \\
\text { discriminati } \\
\text { on }\end{array}$ & $\begin{array}{l}\text { Also termed 'Net Neutrality'. All } \\
\text { communication over the network should be } \\
\text { treated equally. }\end{array}$ \\
\hline $\begin{array}{l}\text { Bottom-up } \\
\text { design }\end{array}$ & $\begin{array}{l}\text { Design and development are done openly. } \\
\text { The community are actively encouraged to } \\
\text { participate. }\end{array}$ \\
\hline Universality & $\begin{array}{l}\text { All hardware must be able to communicate } \\
\text { with each other to facilitate people sharing } \\
\text { information. }\end{array}$ \\
\hline Consensus & $\begin{array}{l}\text { A transparent, participatory process to } \\
\text { create universal standards. }\end{array}$ \\
\hline
\end{tabular}

\section{Table 1: World Wide Web Ideals [42]}

Early internet communities established participatory ideals that are still practised today and have spread outside the IT sector (See table 1). These ideals have been credited as forming the basis for open data in politics, science, education and culture [42]. They are based on the concepts of stakeholder involvement and participatory design, moving away from centralised control and encouraging community involvement in design, development, and decision-making

\section{Research Design}

An in-depth case study [cf. 43] was selected as the most appropriate approach for our research as it supports the investigation of environments in which there are contested meanings, and for studying nonlinear, fragmented, and multi-dimensional phenomena. Our case study centres on the global citizen science project 'We, the Internet' (https://wetheinternet.org/), a mixed-methods study of citizens' and stakeholders' attitudes towards the opportunities and challenges provided by the internet, and future developments in this technology. The project is coordinated by Missions Publiques (France) in collaboration with national organisers across the world. These national partners recruited stakeholders in their respective countries and were part of the facilitation team during the online dialogue. In addition, the project has support from public and private strategic partners such as the German Federal Foreign Office, the United Nations, European Commission, World Economic Forum, Wikimedia Foundation, Internet Society and Google. The strategic partners constituted the advisory board and scientific committee to provide conceptual and scientific guidance. The network of partners is essential in ensuring global outreach with a diversity of participants, as well as enhancing the impact on decision-making processes. This feeds into the key aim of citizen science, to democratise research and enable citizens to impact policy-making. Figure 1 illustrates the timeline.

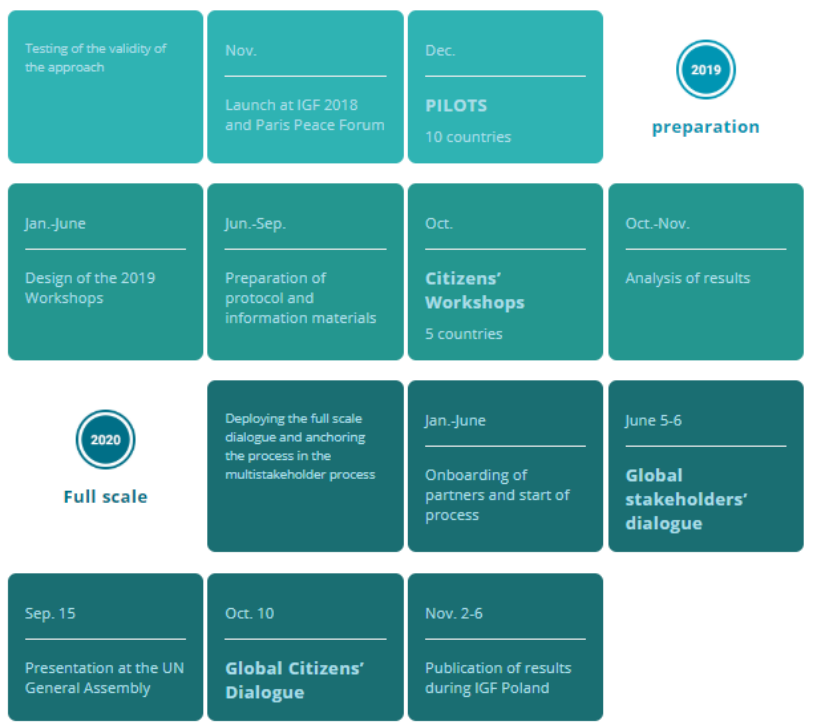

Figure 1: Project Timeline 
The initial concept of We, the Internet was designed around two pillars: a citizen dialogue discussing digital identity, digital public sphere, and artificial intelligence; and a stakeholder dialogue which centred around the future of internet governance. Both dialogues were planned to take place simultaneously in face-to-face meetings on June $6^{\text {th. }}$ However, due to the COVID-19 pandemic, the stakeholder track was implemented on June $5^{\text {th }}$ and $6^{\text {th }}$ online, and the follow up dialogue was postponed to October.

The stakeholder dialogue builds on the comprehensive global initiative set out by The United Nations with the High-Level Panel on Digital Cooperation. In June 2019, the Panel published their report "The Age of Digital Interdependence" and with it, a series of recommendations to improve digital cooperation. We, the Internet, used the process and infrastructure to initiate parallel, but independent stakeholder discussions on Digital Cooperation.

\subsection{Data Collection and Data Analysis}

On 5 and 6 June 2020, Missions Publiques, with the support of a broad coalition of partners worldwide, held a series of four online dialogues tackling the challenges of internet governance. Stakeholders from more than 80 countries around the world came together during threehour online sessions. They discussed the three models proposed by the High-Level Panel of Digital Cooperation launched by Antonio Guterres, SecretaryGeneral of the United Nations [44]. To enhance the deliberation, participants were gathered in subgroups of 3 to 6 stakeholders, plus two facilitators. Discussions in subgroups were conducted in English, French or Spanish, according to the stakeholders' preference.

An open registration process was utilised to ensure a diverse geographical spread among participants, with representatives across six continents Asia, Africa, Europe, North and South America, and Australia. 39.2\% of participants were female, $55.7 \%$ were male, and $5.2 \%$ preferred not to say. In terms of age breakdown, $12.4 \%$ were under 25 years, $32 \%$ were $26-35$ years, $22.7 \%$ were $36-45$ years, $16.5 \%$ were $46-55$ years, $13.4 \%$ were more than 55 years, while $3.1 \%$ preferred not to say.

Data was collected and analysed through a collection of open science practices which are detailed in Table 2. In terms of data collection, a fundamental principle of the deliberation was facilitating an informed discussion among participants. Therefore, participants to the deliberation were sent open access material beforehand, so that each participant was able to have a similar level of knowledge. This material was essential to enable a qualified debate on such a complex issue, as one participant expressed: "What really caught my attention was the way the different internet governance models were presented, with a concise and clear explanation of their scope." (participant from Argentina).

\begin{tabular}{|c|c|}
\hline Practice & Description \\
\hline $\begin{array}{l}\text { Open } \\
\text { registration }\end{array}$ & $\begin{array}{l}\text { An online platform matched registered } \\
\text { volunteers with available discussion } \\
\text { sessions. This supported our sampling } \\
\text { strategy which aimed at global coverage } \\
\text { across countries. Volunteers did not require } \\
\text { expertise on the topic to register, and the } \\
\text { final sample had representation for } \\
\text { different demographics e.g. levels of } \\
\text { internet connectivity. }\end{array}$ \\
\hline $\begin{array}{l}\text { Open access } \\
\text { resources }\end{array}$ & $\begin{array}{l}\text { Resources were provided to volunteers } \\
\text { before the dialogue through a centralised } \\
\text { repository. These resources offered } \\
\text { supporting background information which } \\
\text { helped ensure that volunteers were well- } \\
\text { prepared to provide informed responses } \\
\text { during the dialogue. }\end{array}$ \\
\hline $\begin{array}{l}\text { World Café } \\
\text { Method }\end{array}$ & $\begin{array}{l}\text { The views of volunteers were collected } \\
\text { through a set of structured steps during } \\
\text { roundtable discussions. Facilitators were } \\
\text { present who worked with volunteers as } \\
\text { partners to generate insights into the } \\
\text { effectiveness of potential future } \\
\text { governance models using questionnaires. }\end{array}$ \\
\hline $\begin{array}{l}\text { Stakeholder } \\
\text {-led } \\
\text { evaluations }\end{array}$ & $\begin{array}{l}\text { Data analysis was driven by volunteers } \\
\text { who evaluated proposed internet } \\
\text { governance solutions put forward by } \\
\text { experts / scientists e.g. models of } \\
\text { distributed governance. Participants' views } \\
\text { were collated, aggregated, and then } \\
\text { analysed by the experts. }\end{array}$ \\
\hline $\begin{array}{l}\text { Open access } \\
\text { publications }\end{array}$ & $\begin{array}{l}\text { The final report was published on the } \\
\text { project website, accessible by all. The } \\
\text { results were approved and disseminated by } \\
\text { the German Federal Foreign Office with } \\
\text { the aim of influencing policymaking going } \\
\text { forward. All peer-reviewed articles } \\
\text { delivered by the research team will be open } \\
\text { access on Zenodo. }\end{array}$ \\
\hline
\end{tabular}

Table 2: Adopted Open Science Practices

In order to assess pre-existing opinions, We, the Internet provided balanced briefing material as well as a questionnaire ahead of the dialogue. A World Café Method was used to divide participants into small subgroups to facilitate the deliberation. In each group, a facilitator moderated the discussion while a note-taker recorded key points within worksheets for the analysis. The sub-groups encompassed about 5-8 participants each to ensure a trusted atmosphere for everyone to engage in a meaningful deliberation: "I enjoyed [...] the frank discussion in the focus groups on the advantages and disadvantages of each of them. I was surprised by the wide range of participants and appreciated the willingness of colleagues to discuss and share their thoughts and opinions openly and honestly." 
(participant from Trinidad and Tobago). After each session, participants were then asked to fill out surveys. Ninety-seven participants responded.

For data analysis, stakeholder-led evaluations of different models of internet governance was facilitated throughout the discussion. For the analysis, participants used thematic analysis [45] to discuss the produced material and cluster together similar ideas. The facilitators began by continuously rereading the transcribed content from the consultation to generate a set of codes which they judged as meaningful and important to the study in question. Researchers then grouped these initial codes together to form overarching categories of codes which helped organise the content according to similar themes. This process allowed strongly expressed ideas to emerge.

\section{Findings}

This section presents findings from stakeholder-led evaluations of different models of internet governance. Based on the adoption of open science practices described in Table 2, these will be used to derive key actions points for policy-makers going forward.

\subsection{Improved and effective inclusion must be at the heart of internet governance reform}

There was unanimous agreement among participants that internet governance reform needs to be guided by the involvement of diverse stakeholder groups across different sectors of industry and society. Participants noted that reform initiatives must expand to incorporate views of the private sector (both small, medium size enterprises and multinational corporations), governments (in particular from the legislative branch), as well as citizens. They articulated a desire to make the existing Internet Governance Forum (IGF) more than a "civil society chamber" with little implementing power. With such considerations taken into account, participants noted inclusiveness could serve as a "precondition" for good leadership and legitimacy in the system. However, they cautioned that inclusion requires an increase in both quantity and quality.

Another sentiment from stakeholder-led evaluation was to increase the level of dedicated funds available for the Global South to enable their participation in IGF meetings and other relevant fora. New or improved digital formats could also be introduced for marginalised groups to effectively join remotely. This would allow different affected stakeholder groups to be included in the whole decision-making process, from agenda-setting to discussion, and implementation. As stated by a participant from the Ivory Coast "We need more cooperation between key players to reduce the digital divide at all levels. We want a governance system that facilitates dialogue between the different actors of the Internet community in the country.'

However, external and independent evaluation mechanisms were noted as a priority by participants to ensure adequate representation across all stakeholder groups. Participants also noted that the role of the National and Regional Initiatives (NRI) should be strengthened across all levels: local, national, regional.

\subsection{Transparency and guidance are essential in navigating this complex system}

Participants also discussed open access resources and noted the need for increased transparency on governance processes in order to provide systematic guidance for navigating through the various layers and platforms of internet governance. Participants felt it was vital to communicate clear definitions and understanding of roles and relationships, responsibilities, and accountabilities. However, due to different levels of available resources and capacity, they felt it is difficult to ensure a simple entry point for marginalised stakeholders. The motto "keep it simple" was mentioned on several occasions, as the stakeholders acknowledged the high complexity of the governance system. Despite this, there was little discussion on how to reduce this complexity. It was however discussed that entry points should be made more accessible by leveraging the vertical levels of the NRI structure.

Participants asserted the need to define the roles and function of elements in the IGF+ structure. Participants also spoke about raising Digital literacy via capacity building and targeted support: "What is needed in IGF+ is more inclusivity through education. People must be encouraged, from a young age, to be interested in all internet governance issues in school and upwards. Internet Governance doesn't concern only professionals; it concerns everyone, whether they have the internet or not.". In particular, they felt the "observatory/help desk" could be mandated as a proactive facilitator to help navigate the system. Finally, participants noted that in order to enter the governance system, stakeholders need clear procedural rules on the election/selection process of various bodies so that there is an understanding about how to participate meaningfully. The process within the "policy incubator" and how it develops policy proposals was also said to need more clarification.

\subsection{Trustworthy and stable leadership are required for fair coordination}


There was agreement that ensuring transparency is not only crucial for navigating the highly complex internet architecture but is also at the core of increasing and maintaining trust among all stakeholders. In particular, participants noted that coordination efforts must build transparent rules so that stakeholders can rely on fair procedures. Although informal and confidential discussions remain a valid part of international diplomacy, participants cautioned against "behind the scenes" decisions. It was seen as essential that decisions and the decision-making process are open and transparent. To achieve this, participants recommended that the allocation of funding must be open for tracking.

The UN itself generally enjoys a high level of trust among stakeholders, and the IGF has proven to be a reliable forum for internet governance discussion. Initiatives can build on existing and trustworthy institutions. The IGF+, with a continued UN mandate, can build on this trust by enhancing effective and stable leadership. However, transparent and clear rules were still noted as necessary by participants, with established rotations within the secretariat. This would ensure that everyone can have a fair share in taking up important roles: "Having some clear rules about rotation in positions of the secretariat and other relevant organs would weaken the arguments of digital colonisation."

\subsection{Strengthen coordination and cooperation between stakeholders and different bodies}

There was an understanding among participants of the already very high number of existing fora and discussion groups as well as the complexity of the overall internet governance structure(s). Thus, discussions noted that introducing new platforms must be considered carefully and only introduced if effective and in support of better coordination: "There are so many actors, with so many different interests and all from very different parts of the world, with very different cultures. Despite all these differences, we try to cooperate to find common values, which is difficult, to say the least." Overcoming the divide between technical knowledge and policy and process expertise was said to be critical. In particular, internet architecture was said to call for a strong global moderation.

Participants noted that this requires an open communication channel between NRIs at country level and the "policy incubator" to improve national policies. Panels must be re-organised around current or emerging specific issues instead of broad areas of work to produce more targeted solutions. One suggestion for doing this recommended by participants would be to introduce a two-step approach in which tech and policy community discuss specific challenges individually and then come together to develop joint policy solutions. However, participants asserted that this must ensure a diverse range of stakeholders in this Advisory Group to facilitate a holistic approach.

It was also noted that global vision is needed to build consensus and generate support for needed policies. However, concerns were raised that leaders must establish a "regulated involvement" for the private sector (also at national level) as it is the source of technical innovation and funding and also the direct channel to the end-users of digital products/services.

\subsection{The right resources must be allocated fairly way is key to an impactful digital cooperation}

According to participants, the key to unlocking the potential for improved digital cooperation is the delivery of adequate and sustained funding. A majority $(60 \%)$ of participants see the current IGF trust fund as a useful mechanism that needs to be increased. This was encapsulated by one action point raised by participants "Strengthen the trust fund as the vehicle for funding". Participants also believed that increased funding contributions from large companies was required. The participants debated interesting issues such as the potency and responsibility of the private sector and also the risk of undue influence. However, they conceded that this is an area which requires further research.

However, beyond the question of the amount of funding, a critical question remained around its distribution. Participants identified funding distribution as a key gap on the road to improved digital cooperation. They noted the need to have a transparent and fair distribution of funds between the Global activities and secretariat, and the local and national initiatives. To quote one participant from Africa: "So, if the funding is there and adequate and equitable representation is secured, then we need to have the regional and national IGF strengthened which serves as backbone".

\section{Discussion}

This section provides a discussion concerning our research question: How can open science practices be used to explore socio-political issues of public concern? To answer this question, we presented a citizen science study of stakeholders' attitudes towards the opportunities and challenges provided by the internet, and future developments in this technology. Our paper focuses on the online dialogue, which adopted open science practices such as open registration processes, stakeholder-led evaluations, and open access materials.

Our findings showcase how open science is a key means to mobilise citizens to create data that influences policy and increases political engagement even among marginalised citizens. The importance of results from 
the dialogue is that they will also influence models of internet governance going forward ensuring that the interests, and concerns of stakeholders are represented in future discourse. The results will be presented to strategic partners including United Nations, World Economic Forum, and UNESCO which can help combat public scepticism of science by building long-term relationships between citizens, research, and policy.

The purpose of this process is to make recommendations emerge and then transmit them to the political authorities. It is not only about deliberating for the sake of deliberating; the aim is furthermore to improve decision-making and governance. Findings from the Stakeholder Dialogue will now be integrated as one of many contributions collected by the German Government over the past half-year as part of the HighLevel Panel's follow-up process. Germany is one of the three co-champions in charge of delivering an options paper on the Future of Internet Governance to the UN. More broadly, the results of the Dialogue will feed into the process of the Roadmap on Digital Cooperation issued by the UN Secretary General's (UNSG) Office. The results of the dialogue are well aligned with the Roadmap presented by the Office of the UNSG [44]. Results from the formal multi-stakeholder roundtables were incorporated in the official options paper on the Future of Digital Cooperation.

Indeed the internet was founded on the ideals of openness and transparency which makes it the ideal testing ground for exploring the applicability of open science practices for issues of public concern $[14,16]$. The father of the world wide web, Sir Tim Berners-Lee, is a vocal proponent of open data and open government. He has promoted open government worldwide and cofounded the Open Data Institute (ODI) in 2012 [46]. Unfortunately, the ideals of the early internet and the aims of Open Government have yet to be achieved. Two key early ideals of the internet, bottom-up design and consensus [42] are particularly relevant to our study. Bottom-up design encourages community involvement in design and development [36, 37, 38, 39], while consensus aims to create standards through a transparent, participatory process [33]. Both ideals are relevant to the aim of using input from diverse stakeholders to build a better governance structure.

However, online censorship and digital surveillance are tools employed by governments which limit the collective action potential of open science [47]. Governments engage in surveillance and censorship for commercial reasons; for example, economies heavily invested in the knowledge-producing sectors will work to restrict citizens access to information to promote IP generation [48]. In an open letter on the 28th birthday of the worldwide web, Sir Tim Berners-Lee highlighted the danger posed by companies and governments working together "watching our every move online, and passing extreme laws that trample on our rights to privacy"[49]. Similar to censorship, surveillance creates an imbalance in power between the watcher and the watched. This imbalance is incompatible with debate, census $[47,50]$, and democracy. It's estimated that $71 \%$ of those with internet access live in countries where they can be imprisoned for posting content on political, social, or religious issues [51]. Academics have also signalled that their academic freedoms are being compromised by online censorship and surveillance [52].

To enable new forms of public action, it is necessary to see the emergence of a "deliberative imperative", as epitomised by the pragmatic school of thought in open science. According to French sociologists, Loïs Blondiaux and Yves Sintomer, the success of the "deliberative democracy" studies coincides with the spreading of deliberation and a growing number of deliberative and participative institutions in the political action sphere. These "democratic innovations" mostly follow a deliberative ideal such as defined by Habermas [33], informed groups deliberate together to formulate, in a rational way, concrete solutions by seeking the best decision for the community. The stakeholders' and citizens' dialogues are one of these mechanisms that aim to give different groups an active place in the definition of public policies. Such a mechanism intends to bring together stakeholders around issues of general interest that concern them so that they can take up political issues and debate them collectively. These issues may be directly related to their daily lives or longer-term social issues.

'We, the Internet' contributes insights into how open science following the pragmatic school of thought can allow researchers towards engaged scholarship. It builds on the ethos that collective intelligence emerges from constructive, non-partisan forums. Proposals on internet governance centre on core elements of the philosophy of openness: inclusiveness, transparency, trust, and cooperation. In this model divergent mind-sets are put aside, and everyone is given a chance to speak out to form enlightened, shared and inspiring viewpoints and recommendations for decision-makers.

Our participants expressed their willingness to engage in open science practices going forward, with $92 \%$ stating that they would continue their engagement or recommend their friends/colleagues to participate in such a dialogue. Feedback and statements during the stakeholder dialogue were anonymised, but in post interviews, participants have expressed their motivations to join the deliberation and their lessons learned. On their experience, one participant from Argentina explained their motivation for joining: "I participated in the dialogue to learn about different 
perspectives and ideas for the future of Internet Governance. I was not only able to get my ideas across, but they were also enriched by listening to other colleagues from all over the world."

\section{Conclusion}

In this paper, we discussed the pragmatic school of thought in open science and its applicability to exploring the issues of public concern, such as the future of internet governance. In terms of contributions, we discuss how open science practices were leveraged to derive insights into five emergent themes on the future of internet governance. This first stage of the WTI project has shown that open dialogues can create impact in the political sphere by providing critical input for the future of internet governance. Moving into the next phase, comprehensive citizens' deliberations at a global scale will enable a broader discussion on controversial issues of our time (the (mis)usage of data, disinformation, and the ethics of artificial intelligence). Here the open science approach will be applied to address issues of public concern that significantly expands the insights, attitudes, and opinions over a traditionally more "closed" approach. Future research will contribute insights into the potential of open science to foster change - beginning at the individual level, and moving through groups to eventually support societal level acceptance. In terms of practical contributions, we provided an account of how IS researchers might support openness by engaging diverse stakeholder groups on socio-political issues.

One limitation of the paper is that the case study was primarily focused on the initial stages of engaging stakeholders in dialogue around current challenges. As a result, an in-depth study of potential solutions and the impact derived from the project outcomes on the future development of internet governance was outside the scope of our paper. Future studies can seek to provide a longitudinal analysis of the impact of open science on issues of public concern. The analysis and evaluation of the next stage of the "We, the Internet" project offers such an opportunity. As briefly outlined in Section 3, citizens' assemblies will be held on October $10^{\text {th }}$ around a broad range of internet topics in over 80 countries simultaneously, with an estimated participation of about 100 citizens per country. Research on this international process can provide valuable insights into the impulse for transformative change, beginning from how an open and inclusive deliberative process affects the attitudes of individuals, to impact the collective, non-expert recommendations for global public discourse and political decision-making. The continued analysis of this and other case studies are vital elements for a better understanding of complex issues, such as the interaction between open multi-stakeholder dialogues and the ethics and governance of the internet going forward.

\section{References}

[1] M. Glassman and M. J. Kang, "Pragmatism, connectionism and the internet: A mind's perfect storm", Computers in Human Behavior, 26 (2010), pp. 1412-1418.

[2] Statista, Global digital population as of April 2020, 2020. [3] S. Choudhury and S. Pattnaik, "Emerging themes in elearning: A review from the stakeholders' perspective", Computers \& Education, 144 (2020), pp. 103657.

[4] L. Dahlander and D. M. Gann, "How open is innovation?", Research policy, 39 (2010), pp. 699-709.

[5] H. W. Chesbrough, Open innovation: The new imperative for creating and profiting from technology, Harvard Business Press, 2006.

[6] M. Lasek and S. Jessa, "CHATBOTS FOR CUSTOMER SERVICE ON HOTELS'WEBSITES", Information Systems in Management, 2 (2013), pp. 146-158.

[7] T. H. Davenport and W. J. Glover, "Artificial intelligence and the augmentation of health care decision-making", NEJM Catalyst, 4 (2018).

[8] R. Torres, N. Gerhart and A. Negahban, "Epistemology in the era of fake news: An exploration of information verification behaviors among social networking site users", ACM SIGMIS Database: The DATABASE for Advances in Information Systems, 49 (2018), pp. 78-97.

[9] S. Laato, A. N. Islam, M. N. Islam and E. Whelan, "What drives unverified information sharing and cyberchondria during the COVID-19 pandemic?", European Journal of Information Systems (2020), pp. 1-18.

[10] S. Zuboff, The Age of Surveillance Capitalism: The Fight for a Human Future at the New Frontier of Power, Profile Books, 2019.

[11] G. Walsham, "Are we making a better world with ICTs? Reflections on a future agenda for the IS field", Journal of Information Technology, 27 (2012), pp. 87-93.

[12] M. Carter and V. Grover, "Me, My Self, and I (T): Conceptualizing Information Technology Identity and its Implications", Mis Quarterly, 39 (2015).

[13] B. C. Stahl, "Responsible research and innovation in information systems", European Journal of Information Systems, 21 (2012), pp. 207-221.

[14] S. Bartling and S. Friesike, Opening science: The evolving guide on how the internet is changing research, collaboration and scholarly publishing, Springer Nature, 2014.

[15] S. Mazumdar, L. Ceccaroni, J. Piera, F. Hölker, A. Berre, R. Arlinghaus and A. Bowser, Citizen science technologies and new opportunities for participation, UCL Press, 2018.

[16] European Commission, Open Innovation, Open Science, Open to the World - A vision for Europe, Publications of the European Union, Luxembourg, 2016. 
[17] OECD, "Making open science a reality: " OECD Science, Technology and Industry Policy Papers (2015), pp. 25.

[18] M. Nielsen, Reinventing discovery: the new era of networked science, Princeton University Press, 2020.

[19] L. Z. Karvalics and L. Juhász, Teaching Social Informatics for Engineering Students, Springer US, Boston, MA, 2006, pp. 65-72.

[20] P. A. Busch and S. McCarthy, "Antecedents and consequences of problematic smartphone use: A systematic literature review of an emerging research area", Computers in Human Behavior (2020), pp. 106414.

[21] Oxford Dictionary, Definition of openness in English, Oxford Living Dictionaries, 2017.

[22] D. Riehle, J. Ellenberger, T. Menahem, B. Mikhailovski, Y. Natchetoi, B. Naveh and T. Odenwald, "Open collaboration within corporations using software forges", IEEE software, 26 (2009), pp. 52-58.

[23] M. Janssen, Y. Charalabidis and A. Zuiderwijk, "Benefits, adoption barriers and myths of open data and open government", Information systems management, 29 (2012), pp. 258-268.

[24] J. Feller and B. Fitzgerald, Understanding open source software development, Addison-Wesley London, 2002.

[25] E. v. Hippel and G. v. Krogh, "Open source software and the "private-collective" innovation model: Issues for organization science", Organization science, 14 (2003), pp. 209-223.

[26] O. Tacke, Open Science 2.0: how research and education can benefit from open innovation and Web 2.0, On collective intelligence, Springer, 2010, pp. 37-48.

[27] S. Hecker, M. Haklay, A. Bowser, Z. Makuch, J. Vogel and A. Bonn, "Innovation in open science, society and policy-setting the agenda for citizen science", Citizen science: innovation in open science, society and policy. UCL Press, London, UK (2018), pp. 1-23.

[28] H. Riesch, C. Potter and L. Davies, "What is public engagement, and what is it for? A study of scientists' and science communicators' views", Bulletin of Science, Technology \& Society, 36 (2016), pp. 179-189.

[29] J. P. Holdren, Addressing Societal and Scientific Challenges through Citizen Science and Crowdsourcing. Memorandum to the heads of executive departments and agencies. , in O. o. s. a. t. policy, ed., Washington, D.C. 20502. , 2015.

[30] A. Wiggins and K. Crowston, From Conservation to Crowdsourcing: A Typology of Citizen Science, 2011 44th Hawaii International Conference on System Sciences, 2011, pp. 1-10.

[31] C. Kullenberg and D. Kasperowski, "What Is Citizen Science? - A Scientometric Meta-Analysis", PLOS ONE, 11 (2016), pp. e0147152.

[32] F. Heigl, B. Kieslinger, K. T. Paul, J. Uhlik and D. Dörler, "Opinion: Toward an international definition of citizen science", Proceedings of the National Academy of Sciences, 116 (2019), pp. 8089.

[33] J. Habermas, The Theory of Communicative Action: Reason and the rationalization of society, Beacon Press, 1984.
[34] B. Manin, E. Stein and J. Mansbridge, "On Legitimacy and Political Deliberation", Political Theory, 15 (1987), pp. 338-368.

[35] EC, Science with and for Society, Policy: Public Engagement European Commission, 2013.

[36] E. Bjögvinsson, P. Ehn and P.-A. Hillgren, "Design Things and Design Thinking: Contemporary Participatory Design Challenges", Design Issues, 28 (2012), pp. 101-116. [37] M. J. Muller and S. Kuhn, "Participatory design", Commun. ACM, 36 (1993), pp. 24-28.

[38] F. Kensing and J. Blomberg, "Participatory design: Issues and concerns", Computer Supported Cooperative Work (CSCW), 7 (1998), pp. 167-185.

[39] V. Farrell, G. Farrell, K. Mouzakis, C. Pilgrim and P. Byrt, PICTIOL: a case study in participatory design, Proceedings of the 18th Australia conference on ComputerHuman Interaction: Design: Activities, Artefacts and Environments, Association for Computing Machinery, Sydney, Australia, 2006, pp. 191-198.

[40] M. van der Velden and C. Mörtberg, Participatory Design and Design for Values, in J. van den Hoven, P. E. Vermaas and I. van de Poel, eds., Handbook of Ethics, Values, and Technological Design: Sources, Theory, Values and Application Domains, Springer Netherlands, Dordrecht, 2021, pp. 1-22.

[41] P. Dalsgaard, "Participatory Design in Large-Scale Public Projects: Challenges and Opportunities", Design Issues, 28 (2012), pp. 34-47.

[42] WWWF, History of the Web, World Wide Web Foundation, 2020.

[43] P. Darke, G. Shanks and M. Broadbent, "Successfully completing case study research: combining rigour, relevance and pragmatism", Information systems journal, 8 (1998), pp. 273-289.

[44] A. Guterres, Report of the Secretary-General Roadmap for Digital Cooperation, United Nations, 2020, pp. 39.

[45] M. Q. Patton, Qualitative research and evaluation methods, Sage, Thousand Oaks, CA, 2002.

[46] ODI, Sir Tim Berners-Lee: Inventor of the web and President and Co-Founder of the Open Data Institute, 2020.

[47] E. Stoycheff, G. S. Burgess and M. C. Martucci, "Online censorship and digital surveillance: the relationship between suppression technologies and democratization across countries", Information, Communication \& Society, 23 (2020), pp. 474-490.

[48] S. A. Meserve and D. Pemstein, "Google Politics: The Political Determinants of Internet Censorship in

Democracies", Political Science Research and Methods, 6 (2018), pp. 245-263.

[49] T. Berners-Lee, Three challenges for the web, according to its inventor, Web Foundation, 2017.

[50] S. Robbins and A. Henschke, "The Value of Transparency: Bulk Data and Authoritarianism", Surveillence and Society, 15 (2017), pp. 582-589.

[51] A. Shahbaz and A. Funk, Freedom on the Net 2019: The Crisis of Social Media, Freedom House, 2019.

[52] L. M. Tanczer, R. J. Deibert, D. Bigo, M. I. Franklin, L. Melgaço, D. Lyon, B. Kazansky and S. Milan, "Online Surveillance, Censorship, and Encryption in Academia", International Studies Perspectives, 21 (2019), pp. 1-36. 